\title{
Percutaneous Mesh Expansion and Fixation at the Retro-Rectus Plane without Stabs by Using Redirecting Suture Hook in Midline Hernias Repair
}

\author{
Ahmed E. Lasheen, Alaa N. El Sadek, Adel M. Tolba, Emad Salah, Ayman F. Mehanna \\ General Surgery Department, Faculty of Medicine, Zagazig University, Zagazig, Egypt \\ E-mail: leniem@gmail.com,tgkim@chonnam.ac.kr \\ Received December 27, 2010; revised April 10, 2011; accepted April 21, 2011
}

\begin{abstract}
Background: Mesh expansion and fixation at retro-rectus plane through multiples stabs produces good results. But these stabs cause cosmetic disorders for the patients and doctors. So, we find some modification to do this procedure without these stabbing wounds in midline hernial repair. Patients and methods: This technique was used to fix the mesh at retro-rectus plane in 50 patients suffering from midline hernias, from January 2008 through January 2010 at Zagazig university Hospital, Egypt. Laparotomy incision was done over the hernial sac or at old incision; the contents were then released and reduced into peritoneal cavity without much subcutaneous dissection. The suitable sheet of polypropylene mesh to cover the hernial defect and any weak area was prepared and fixed at retro-rectus plane percutaneously without stabbing wounds by using redirecting suture hook. The mean period of follow up was 26 months. Results: There was no recurrence during the period of follow up. Five patients developed subcutaneous bluish discoloration at the site of some stitches, which disappear within two weeks with conservative treatment. Conclusion: Percutaneous mesh expansion and fixation at retro-rectus plane by using redirecting suture hook procedure has good results in recurrence rate and cosmetic appearance.
\end{abstract}

Keywords: Percutaneous Fixation, Retro-Rectus Mesh, Redirecting Suture Hook

\section{Introduction}

The repair of incisional and ventral hernias continues to be a surgical challenge. Reports published in the medical literature indicate $3 \%$ to $13 \%$ of laparotomy patients develop incisional hernias. [1] Moreover, clinical studies indicate that the traditional, or open technique to repair large abdominal wall defects is associated with recurrence rates ranging from 25\% - 49\%. [2,3] Multiple studies have documented that traditional open ventral hernioplasty has significant complication as infection, hematoma, seroma, chronic sinus tract formation, mesh extrusion, fistula formation and non healing wound. [4] In addition, the wide dissection of soft tissue that is required for a Stoppa type retro-rectus repair or a Cherrel type anterior repair leads to the many wound related problems. [5] The stabbing technique for mesh expansion and fixation at retro-rectus plane associated with good results and avoided the all wound complications, as this procedure not associated by much soft tissue dissec- tion. [6] But, the multiple stab wounds in the anterior abdominal wall through which every stitch was tied, cause cosmetic disorder for patients and doctors. So, some modification was done in stabbing technique to tie the stitches at anterior plane to the anterior rectus sheath without made these multiple stab wounds. What is called percutaneous mesh expansion and fixation at retro-rectus plane by using redirecting suture hook in midline hernias repair.

\section{Patients and Methods}

Fifty patients with midline hernias were included in this study at the Zagazig University Hospital, Egypt from January 2008 through January 2010. Thirty patients have incisional hernias after midline laparotomy incision and twenty patients have big midline hernias, with divercation recti (37 female and 13 male). Full informed discussion with all patients about the nature of this technique and taken acceptance from every patient were done be- 
fore beginning of the work including their data in this study. The patients ages were ranged from 20 to 56 years (mean age was 42.7 years). All patients were subjected to percutaneous mesh expansion and fixation at retro-rectus plane by using redirecting suture hook. Small incision is placed on hernial sac or in old incision. The sac is dissected and open without much subcutaneous dissection, the sac contents are returned to abdominal cavity after freeing of all adhesion, and peritoneum cavity is closed. The retro-rectus plane is reached by longitudinal incisions on the medial borders of rectus sheaths on both side without subcutaneous dissection. The posterior rectus sheaths on both sides are approximated together by continuous suture using 2/0 polypropylene suture to approximate the two recti and correct divercation. Sheet of polypropylene mesh to cover the hernial defect and any weak area of the anterior abdominal sheath all around by about $5 \mathrm{~cm}$ is prepared and suitable numbers of sutures are placed on the edge of mesh using No. 0 non absorbable suture (polypropylene). The centre of mesh is fixed in midline by using 2/0 polypropylene. The two tip hole needles are passed through the skin of anterior abdominal wall without stab wound to retro-rectus plane and then, came to the main wound to bring the suture strands outside the skin and caught them by artery forceps. After completion of this procedure for all mesh sutures, the expansion and fixation of mesh can be examined, new sutures can be added if needed at this step. The redirecting suture hook [7] is passed from the operation wound at the anterior plane to the anterior rectus sheath to catch the two strands of each suture, and then, it is withdrawn to bring the two suture strands from the operation wound. Then, the two suture strands are tied through the operation wound, where the suture knot lies directly on the anterior abdominal sheath (Figures 1(a)-(e)). The anterior rectus sheaths on both sides are approximated by some stitches and the wound is closed in layers with suction drainage (Figures 2(a)-(e)). Early ambulation was allowed. Oral feeding was allowed 24 hours postoperatively unless the intestinal sounds were absent or there was abdominal distension. The patients were discharged from hospital 6 hours after starting the oral feeding, if there was no vomiting or abdominal distension. The follow up period was ranged from 6 to 30 months (Mean period was 26 months).

\section{Results}

The operation time ranged from 60 to 120 minutes (Mean 90 minutes). The length of the hospital stay averaged 2.3 days (Range 1.5 to 2.5 days). The drainage was removed after 5 to 9 days according to seroma amount comes through it. Five patients show bluish discoloration at the skin corresponding to the site of passage of redirecting suture hook at anterior plane of anterior rectus sheath (Figure 3). This bluish discoloration disappeared with conservative treatment after about 2 weeks. No recurrence or other complications have been recorded during
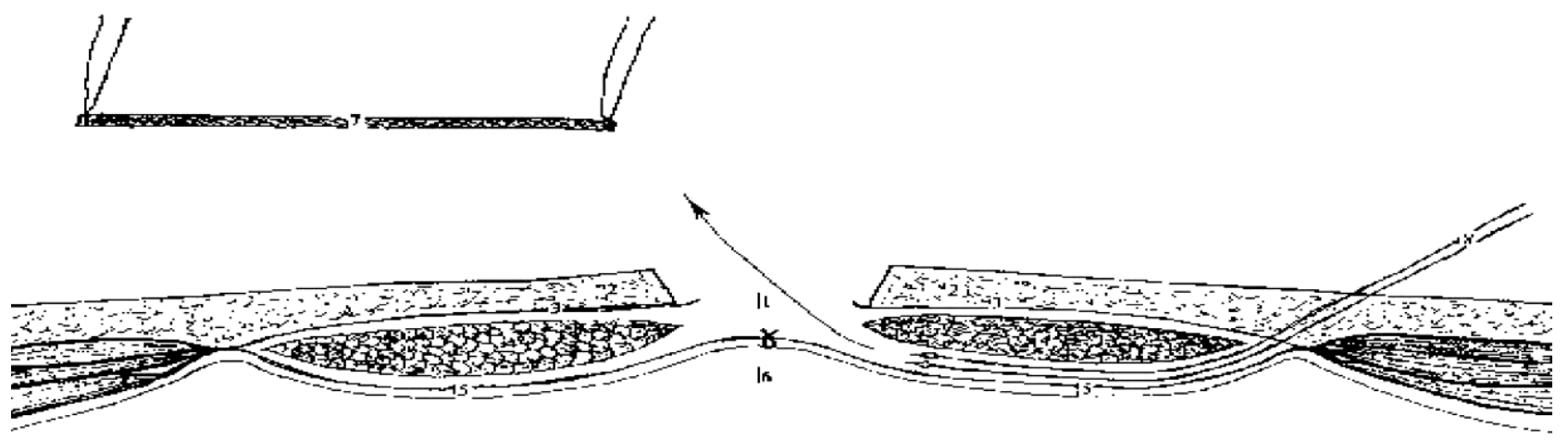

(a)

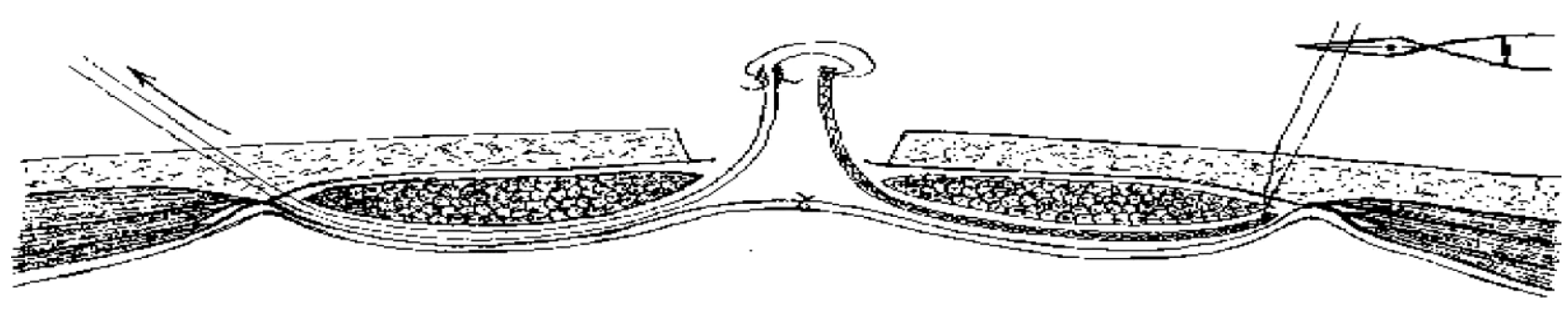

(b) 


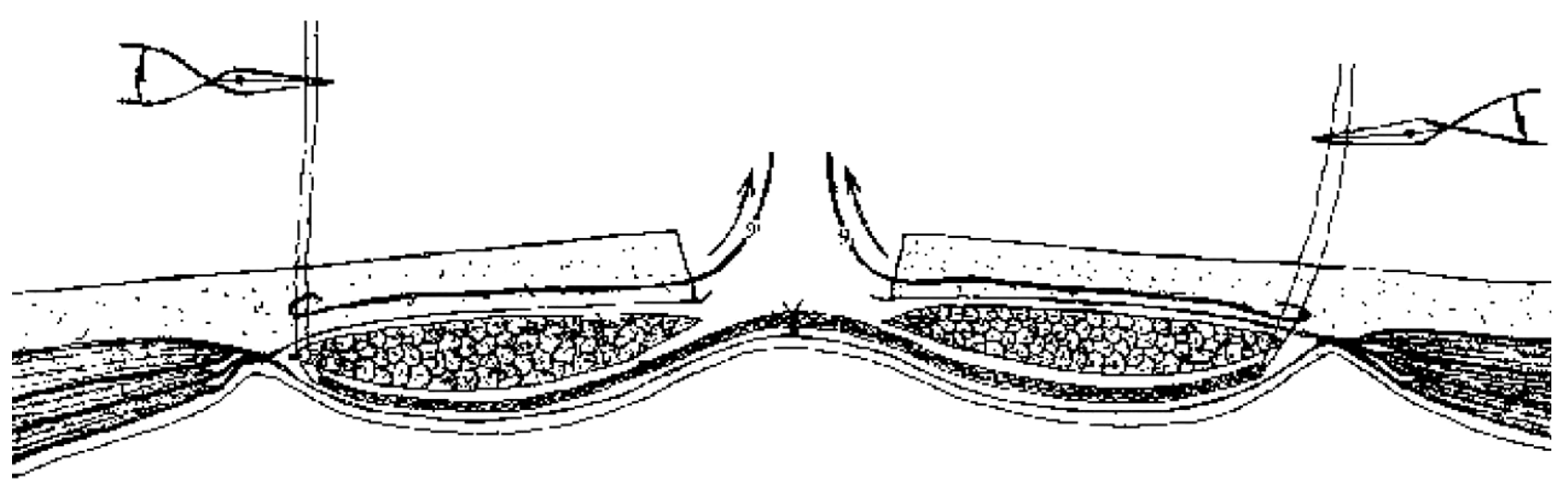

(c)

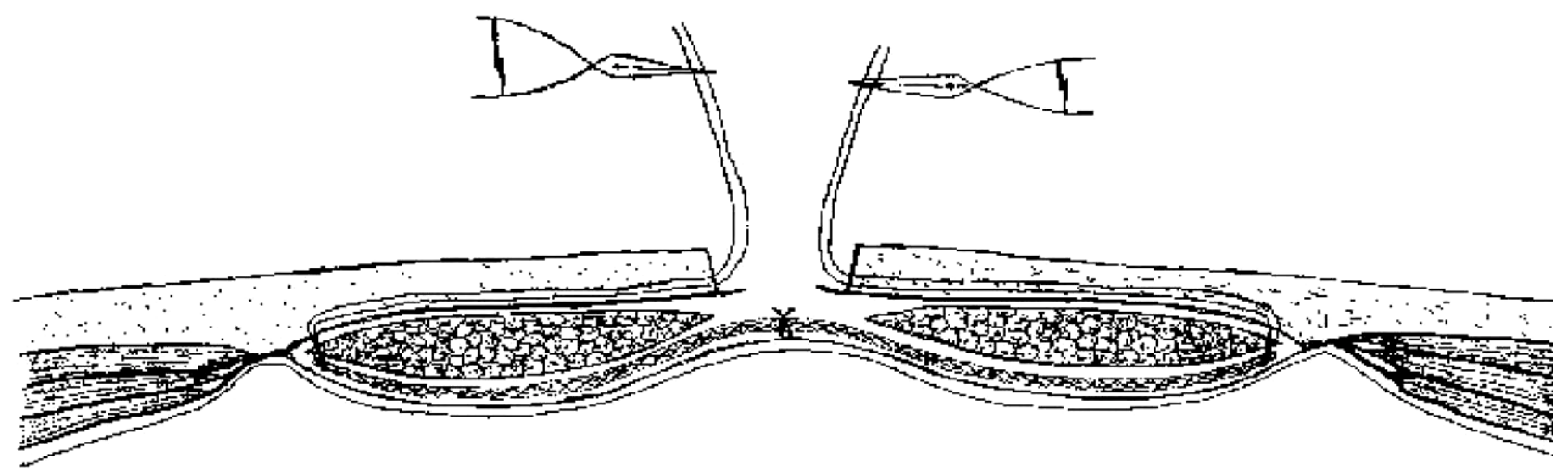

(d)

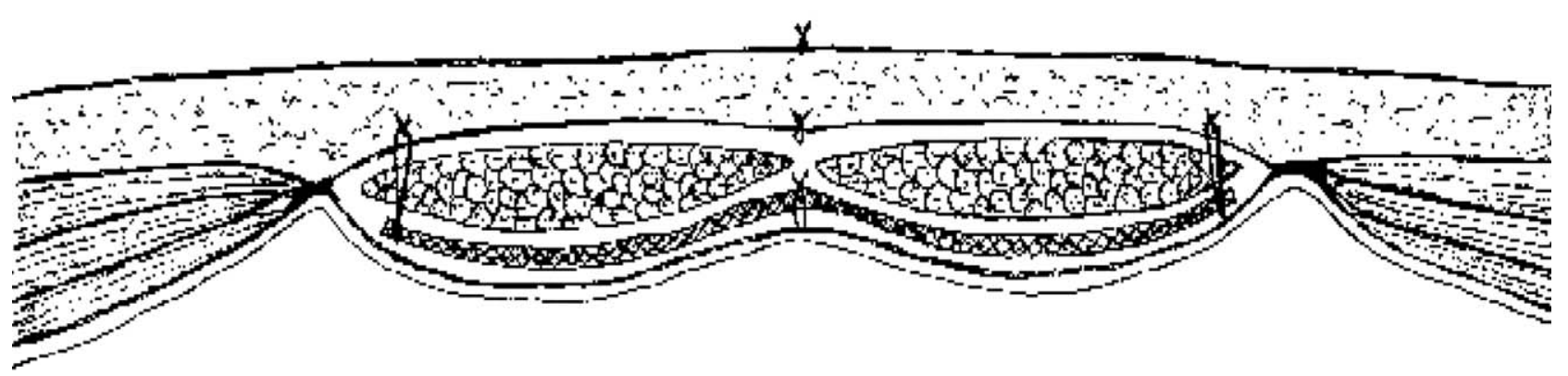

(e)

Figure 1. (a)-(e) Diagram of mesh fixation with redirecting suture hook in midline hernias repair procedure. 1-Main operation wound. 2-Skin and subcutaneous tissue.3-Anterior rectus sheath. 4-Rectus muscle. 5-Posterior rectus sheath. 6-Abdominal cavity. 7-Sheet of polypropylene mesh. 8-Two tip hole needles. 9-Redirecting suture hook. (a) Limited dissection to expose the medial borders of rectus sheath on both sides and two longitudinal incisions were done. Then, continuous suture to approximate posterior layers of rectus sheaths on both sides in midline was done. The suitable mesh in dimensions was prepared and putted suitable number of sutures on its edges. Then, the two tip hole needles passed from skin of anterior abdominal wall to retro-rectal plane to appear from operation wound; (b) The strands of the corresponding suture were fixed to the hole of each needle, then withdrawing the two needles back to bring the suture strands from the skin of anterior abdominal wall. The suture strands were detached from each needle and caught with artery forceps. Then, all the previous steps were return with all mesh sutures to bring each suture strands from corresponding site of anterior abdominal wall; (c) Completion of putting all sutures in their corresponding skin site of anterior abdominal wall was done. Then, examined the mesh fixation and expansion, and can be added new sutures to produce well fixed and expanded mesh. The redirecting suture hook was passed through the operation wound at anterior fascial plane to catch the two strands of suture and withdrawn; (d) All sutures strands were completed to bring from operation wound and caught by artery forceps; (e) All mesh sutures strands were tied through the operation wound and their notes lied directly on anterior rectus sheath. Then, approximation of two anterior rectus sheaths over mesh and wound was closed with drain. 

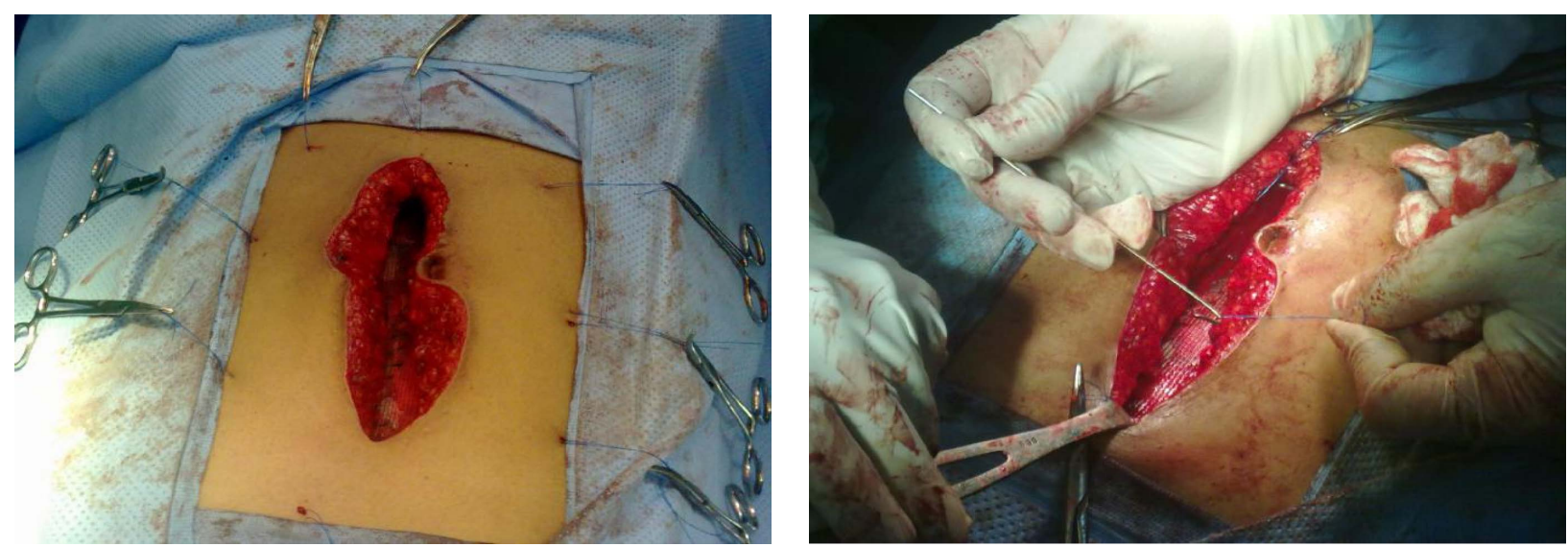

(a) (b)
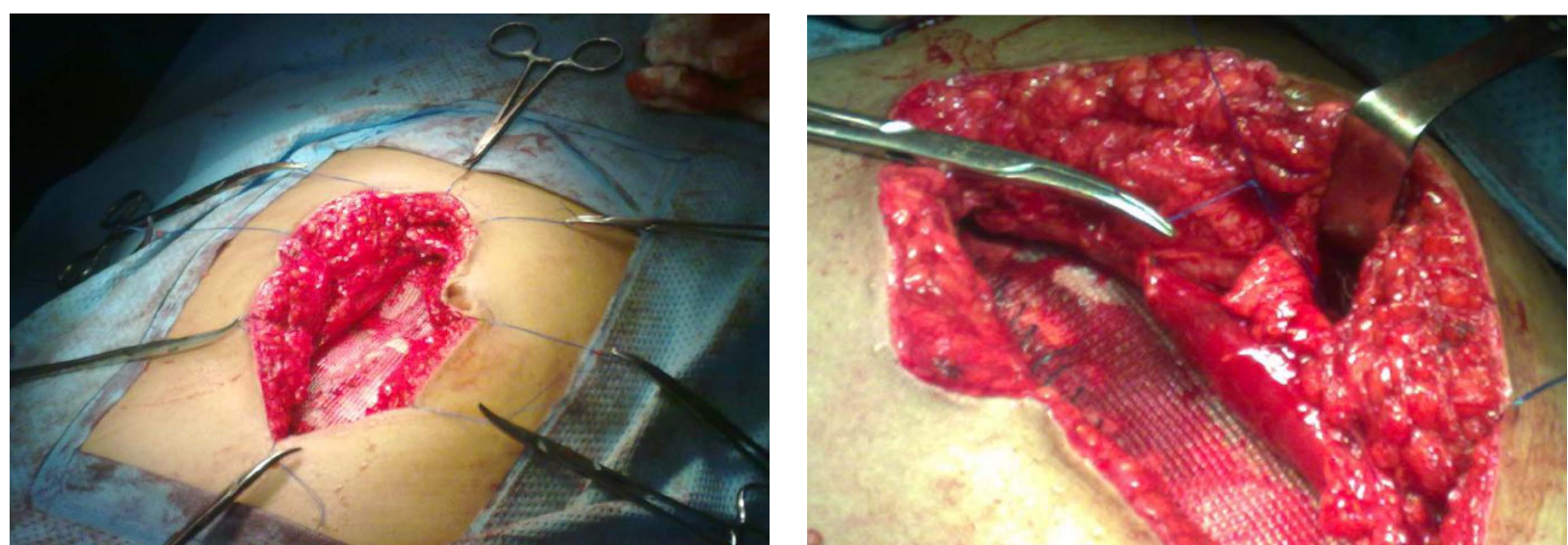

(c)

(d)

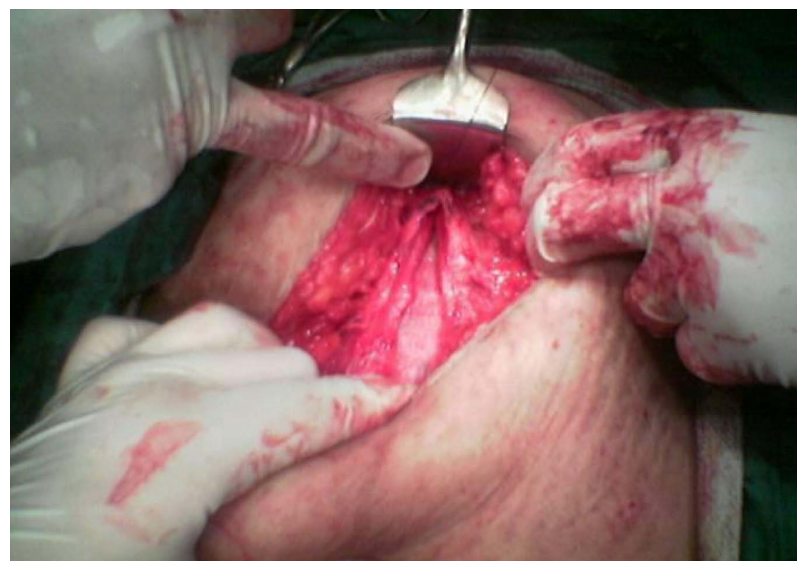

(e)

Figure 2. (a)-(e) Photo of mesh fixation with redirecting suture hook in midline hernias repair procedure. Male patient has midline incisional hernia after exploratory laparotomy, where subjected to this procedure for repair. (a) Finishing of putting all sutures which required producing good expanded and fixed mesh was complete. At first the each suture strands came from the skin of anterior abdominal wall; (b) The redirecting suture hook was passed through the operation wound at anterior fascial plane to catch the two strands of suture and withdrawn to bring them from operation wound; (c) The two strands of each suture were came from the operation wound and caught by artery forceps. (d) Suture strands of each suture were tied through the operation wound and the knot lied directly on the anterior rectus sheath; (e) Closure of the anterior rectus sheaths over the mesh is done. 


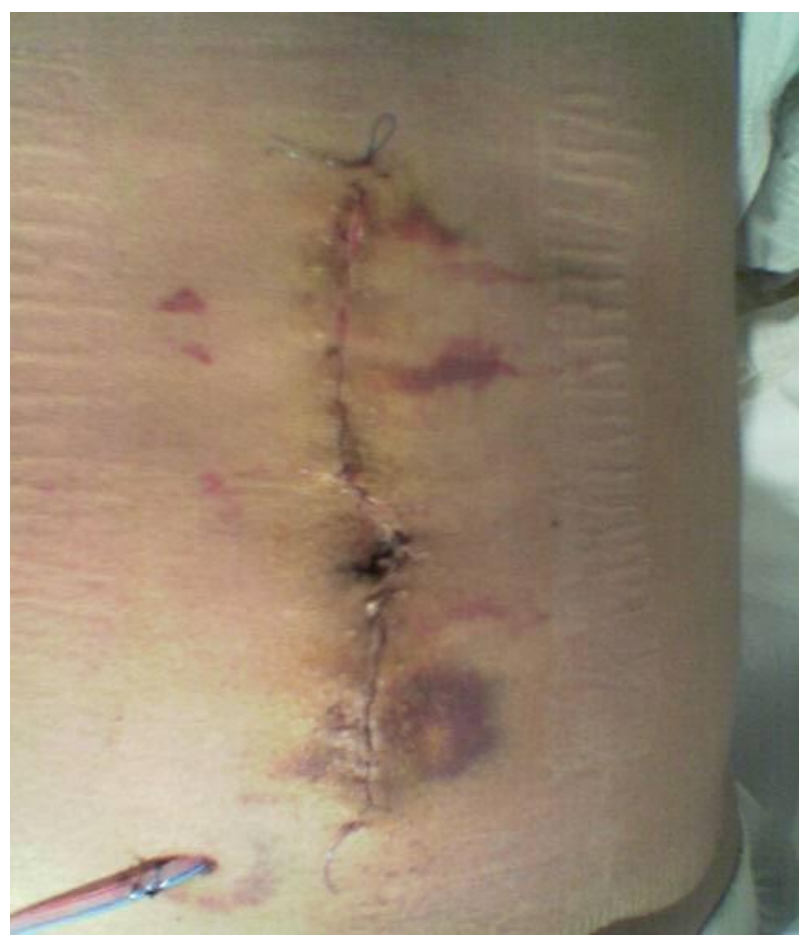

Figure 3. Five patients show bluish discoloration at the skin corresponding to the site of passage of redirecting suture hook at anterior plane of anterior rectus sheath.

the period of follow up.

\section{Discussion}

Incisional hernia will develop in $3 \%$ to $13 \%$ of patients undergoing laparotomy. [8] The introduction of a prosthetic mesh to ensure abdominal wall strength without tension has decreased the recurrence rate to a still significant $12.5 \%$ to $19 \%$. [9] The traditional operation for ventral hernia repair that requires a prosthetic mesh generally necessitates significant soft tissue dissection in tissues that are already of poor quality as well as flap creation, and increasing complication rate of up to $20 \%$. [10] The sublay prosthetic repair technique of midline hernia associated with large incisions and wide area of dissection. [11,12] Recently, there is a dramatic shift toward management of ventral hernia repair by using the laparoscopic technique. Results appear quite promising with lower recurrence rates compared with conventional open surgical repair. Also, hospital stay appears to be shortened and a faster resumption of normal activities. [1] However, there are some difficulties with laparoscopic repair of ventral hernia including scarred abdomen in which, it is impossible to safely introduce pneumoperitoneum, and acute abdomen with possibility of strangulated infarct bowel. In addition, laparoscopic intraperitoneal mesh repair may be associated with injury to sac contents during dissection, adhesion of the mesh to intraperitoneal organs, intestinal fistula formation or intestinal obstruction. [4] The stabbing technique for midline hernias repair carries good results, as a large piece of prosthetic mesh which is placed under the hernial defect with a wide margin of mesh outside the defect at retro-rectus plane. This mesh is anchored into place with suitable number of the sutures and secured to the anterior abdominal wall sheath through small stabs incisions. [7] These stabs incisions cause disfigure scars for patients. In present study, these stabbing incisions were avoided, by bring the two strands of each suture to operative wound by using redirecting suture hook, where they were tied. Our present technique has the same good results of stabbing technique of mesh repair of midline hernia and at the same time avoided the producing multiples stabs wounds.

\section{References}

[1] B. T. Heniford, A. Park, B. J. Ramshaw and G. Voeller, "Laparoscopic Ventral and Incisional Hernia Repair in 407 Patients," Journal of the American College of Surgeons, Vol. 190, No. 6, 2000, pp. 645-650. doi:10.1016/S1072-7515(00)00280-5

[2] V. J. Hesselink, R. W. Luijendijk, J. H. De Wilt, R. Heide and J. Jeekel, "An Evaluation of Risk Factors in Incisional Hernia Recurrence,” Surgery, Gynecology and Obstetrics, Vol. 176, No. 3, 1993, pp. 228-234.

[3] K. A. Leblanc and W.V. Booth, "Laparoscopic Repair of Incisional Abdominal Hernias Using Expanded Polytetrafluoroethylene: Preliminary Findings," Surgical Laparoscopy Endoscopy and Percutaneous, Vol. 3, No. 1, 1993, pp. 39-41.

[4] G. E. Leber, J. L. Garb, A. I. Alexander and W. P. Reed, "Long-Term Complications Associated with Prosthetic Repair of Incisional Hernias,” Archives of Surgery, Vol. 133, No. 4, 1998, pp. 378-382. doi:10.1001/archsurg.133.4.378

[5] T. J. White, M. C. Santos and J. S. Thompson, "Factors Affecting Wound Complications in Repair of Ventral Hernias,” The American Journal of Surgery, Vol. 64, No. 3, 1998, pp. 276-280.

[6] A. E. Lasheen, "Mesh Expansion and Fixation at the Retro-Rectus Plane Through Multiple Stabs by Using Two Tip Hole Needles in Midline Hernias Repair. A Prospective Study," International Journal of Surgery, Vol. 6, No. 5, 2008, pp. 367-370. doi:10.1016/j.ijsu.2008.06.002

[7] A. E. Lasheen, A. Elzeftawy, A. H. Ahmed and W. E. Lotfy, "Anatomical Closure of Trocar Site by Using Tip Hole Needle and Redirecting Suture Hook,” Surgical Endoscopy, Vol. 24, No. 10, 2010, pp. 2637-2639. doi:10.1007/s00464-010-1012-3

[8] M. Mudge and L. E. Hughes, "Incisional Hernia: A 10 Year Prospective Study of Incidence and Attitudes," 
British Journal of Surgery, Vol. 72, No. 1, 1985, pp. 70-71. doi:10.1002/bjs.1800720127

[9] J. J. Bauer, M. T. Harris, I. Kreel and I. M. Gelernt, "Twelve-Year Experience with Expanded Polytetrafluoroethylene in the Repair of Abdominal Wall Defects," Mount Sinai Journal of Medicine, Vol. 66, No. 1, 1999, pp. 20-25.

[10] D. Lomanto, S. G. Iyer, A. Shabbir and W. K. Cheah, "Laparoscopic Versus Open Ventral Hernia Mesh Repair:
A Prospective Study,” Surgical Endoscopy, Vol. 20, No. 7, 2006, pp. 1030-1035. doi:10.1007/s00464-005-0554-2

[11] R. E. Stoppa, "The Treatment of Complicated Groin and Incisional Hernias,” World Journal of Surgery, Vol. 13, No. 5, 1989, pp. 545-554. doi:10.1007/BF01658869

[12] G. E. Wantz, "Incisional Hernioplasty with Mersilene," Surgery, Gynecology and Obstetrics, Vol. 172, No. 2, 1991, pp. 129-137. 